OPEN ACCESS

Edited by:

Longxiang Su,

Peking Union Medical College Hospital (CAMS), China

Reviewed by:

Yanxing Wei,

University of Toronto, Canada

Guodong Fu,

Mount Sinai Hospital, University of

Toronto, Canada

*Correspondence:

Wei Yang

yw8278@hotmail.com; yw8278@hust.edu.cn

Cao Yang

caoyangunion@hust.edu.cn

tThese authors have contributed equally to this work and share first

authorship

Specialty section:

This article was submitted to Infectious Diseases - Surveillance,

Prevention and Treatment,

a section of the journal

Frontiers in Medicine

Received: 20 May 2020

Accepted: 20 July 2020

Published: 06 August 2020

Citation:

Feng X, Li P, Ma L, Liang H, Lei J, Li W, Wang K, Song Y, Li S, Yang W and Yang $C$ (2020) Clinical

Characteristics and Short-Term

Outcomes of Severe Patients With

COVID-19 in Wuhan, China.

Front. Med. 7:491.

doi: 10.3389/fmed.2020.00491

\section{Clinical Characteristics and Short-Term Outcomes of Severe Patients With COVID-19 in Wuhan, China}

\author{
Xiaobo Feng ${ }^{1 \dagger}$, Peiyun $\mathrm{Li}^{2 \dagger}$, Liang Ma ${ }^{1 \dagger}$, Hang Liang ${ }^{1}$, Jie Lei ${ }^{1}$, Wenqiang $\mathrm{Li}^{1}$, Kun Wang ${ }^{1}$, \\ Yu Song ${ }^{1}$, Shuai $\mathrm{Li}^{1}$, Wei Yang ${ }^{3,4 *}$ and Cao Yang ${ }^{1 *}$
}

${ }^{1}$ Department of Orthopaedics, Union Hospital, Tongji Medical College, Huazhong University of Science and Technology, Wuhan, China, ${ }^{2}$ Department of Clinical Nutrition, Wuhan Children's Hospital (Wuhan Maternal and Child Healthcare Hospital), Tongji Medical College, Huazhong University of Science and Technology, Wuhan, China, ${ }^{3}$ Hubei Key Laboratory of Food Nutrition and Safety, Department of Nutrition and Food Hygiene, Tongji Medical College, Huazhong University of Science and Technology, Wuhan, China, ${ }^{4}$ Department of Nutrition and Food Hygiene and MOE Key Lab of Environment and Health, School of Public Health, Tongji Medical College, Huazhong University of Science and Technology, Wuhan, China

Background: A novel pneumonia (COVID-19) spread rapidly throughout worldwide, in December, 2019. Most of the deaths have occurred in severe and critical cases, but information on prognostic risk factors for severely ill patients is incomplete. Further research is urgently needed to guide clinicians, and we therefore prospectively evaluate the clinical outcomes of 114 severely ill patients with COVID-19 for short-term at the Union Hospital in Wuhan, China.

Methods: In this single-centered, prospective, and observational study, we enrolled 114 severely ill patients with confirmed COVID-19 from Jan 23, 2020, to February 22, 2020. Epidemiological, demographic, laboratory, treatment, and outcome data were recorded, and the risk factors for poor outcome were analyzed.

Results: Among the 114 enrolled patients with a mean age of $63.96 \pm 13.41$ years, 94 (82.5\%) patients were classified as a good outcome group. Common clinical manifestations included fever, cough, and fatigue. Compared with the good outcome group, 20 (17.5\%) patients in the poor outcome group more frequently exhibited lymphopenia, and lower levels of albumin, partial arterial oxygen pressure, higher levels of lactate dehydrogenase, creatine kinase, hypersensitive troponin I, C-reactive protein, ferritin, blood urea nitrogen, and D-dimer, as well as markedly higher levels of IL-6 and IL-10. Absolute numbers of T lymphocytes, CD8 + T cells, decreased in almost all the patients and were markedly lower in the poor outcome group than the good outcome group. We also found that traditional Chinese medicine can significantly improve the patient's condition, which is conducive to the transformation from a severe to mild condition. In addition, univariate and multivariate Cox analyses of potential factors for poor outcome patients indicated that cytokine storms and uncontrolled inflammation responses as well as liver, kidney, and cardiac dysfunction are related to the development of a poor outcome. 
Conclusion: In summary, we reported this single-centered, prospective, and observational study for short-term outcome in severe patients with COVID-19. We found that cytokine storms and uncontrolled inflammation responses as well as liver, kidney, and cardiac dysfunction may play important roles in the final outcome of severely ill patients with COVID-19. Our study will allow clinicians to benefit and rapidly estimate the likelihood of a short-term poor outcome for severely ill patients.

Keywords: COVID-19, SARS-CoV-2, severe patients, short-term outcomes, inflammation

\section{INTRODUCTION}

A pneumonia caused by a novel coronavirus, severe acute respiratory syndrome corona virus 2 (SARS-CoV-2), spread rapidly throughout worldwide in December 2019 (1). Despite progress made in our understanding of the characteristics of the disease, there are currently no drugs to combat SARS$\mathrm{CoV}-2$, and patients are primarily provided with supportive treatment. Several studies have indicated that the main symptoms of coronavirus disease 2019 (COVID-19) include fever, cough, and dyspnea (2-5). Huang et al. described the epidemiological, clinical, laboratory, and radiological characteristics of COVID19 , as well as various treatment strategies and outcomes, among 41 patients during the first wave of hospitalizations. They also compared clinical characteristics between patients treated in an intensive care unit (ICU) and those treated in non-ICU (2). Yang et al. also performed a detailed analysis of the patients critically ill with SARS-CoV-2 infection (5). In an analysis of 74 patients with COVID-19 exhibiting gastrointestinal symptoms, Jin et al. suggested that non-classical symptoms have been overlooked, posing a threat to the public (6). Wu et al. further noted that the risk of acute respiratory distress syndrome (ARDS) and death is increased in older adults ( $\geq 65$ years old) with COVID-19 (7). Guo et al. found that diabetes is a risk factor for patients with COVID-19 (8). However, few prospective studies have explored the short-term outcomes of severely ill patients under current medical treatment and the risk factors that affect the shortterm outcomes of severely ill patients, especially pneumonia patients with certain chronic diseases, which accounted for the majority of deaths. Here, we used a single-centered, prospective method to describe the basic clinical characteristics and shortterm outcomes of severe patients in Union hospital, Wuhan, and we further aimed to explore the potential risk factors for poor outcomes among these patients using Cox proportional hazard models.

\section{METHODS}

\section{Study Design and Participants}

This single-center, prospective study included 114 severe patients with confirmed COVID-19 pneumonia hospitalized at the Union Hospital in Wuhan, China, which is a hospital designated to treat patients with COVID-19. From January 23, 2020, to February 22, 2020, we continuously enrolled patients diagnosed with COVID19 based on interim guidance provided by the World Health Organization (WHO). Based on the Diagnosis and Treatment
Scheme for SARS- CoV-2 of Chinese (The Seven Edition), severe patients were diagnosed if one or more of following criteria were met: dyspnea with respiratory rate $(R R) \geq 30$ times/min, resting finger oxygen saturation $\leq 93 \%$, and artery $\mathrm{PaO}_{2} / \mathrm{FiO}_{2} \leq 300 \mathrm{~mm} \mathrm{Hg}(1 \mathrm{~mm} \mathrm{Hg}=0.133 \mathrm{kPa})$. This study was approved by the Ethics Commission of Wuhan Union Hospital of Tongji Medical College, Huazhong University of Science and Technology. Written informed consent was waived due to the emergency of this infectious diseases.

\section{Data Collection}

Data related to clinical characteristics were collected using a case record form modified from the standardized International Severe Acute Respiratory and Emerging Infection Consortium case report form. Epidemiological and demographic data, including age, sex, and coexisting disorders, were also collected. The Baseline laboratory indices and radiographic findings were obtained from clinical electronic medical records. Moreover, the treatment strategies and outcomes were collected until the day of death /discharge or for the first 28 days after a diagnosis of severe illness, whichever was shorter. All missing or vague data, were obtained by communicating with patients and their families. All data were checked by two physicians (Xiaobo Feng and Liang $\mathrm{Ma}$ ), and a third researcher (Wei Yang) adjudicated any difference in interpretation between the two primary reviewers.

\section{Outcomes}

Clinical outcomes after 28 days of consecutive observations were divided into two categories. Patients that had been discharged, those whose condition had been deemed non-severe, and those not requiring mechanical ventilation were considered to have experienced good outcomes. Patients requiring mechanical ventilation and those who had died were considered to have experienced poor outcomes. The criteria for discharge were as follows: normal temperature for more than 3 days $\left(\mathrm{T}<37.3^{\circ} \mathrm{C}\right)$, significant improvement in respiratory symptoms, pulmonary imaging showing significant improvement in acute exudative lesions, and nucleic acid tests negative for respiratory tract specimens such as sputum and nasopharyngeal swabs for two consecutive samplings (at least $24 \mathrm{~h}$ after sampling). Patients with mild clinical symptoms and no signs of pneumonia on radiography were considered to be non-severe. ARDS and shock were confirmed by the WHO guidance for COVID-19. Acute kidney injury was defined according to the serum creatinine. Cardiac injury was identified by the serum concentration of hypersensitive cardiac troponin I (hsTNI) and, if it was above the 


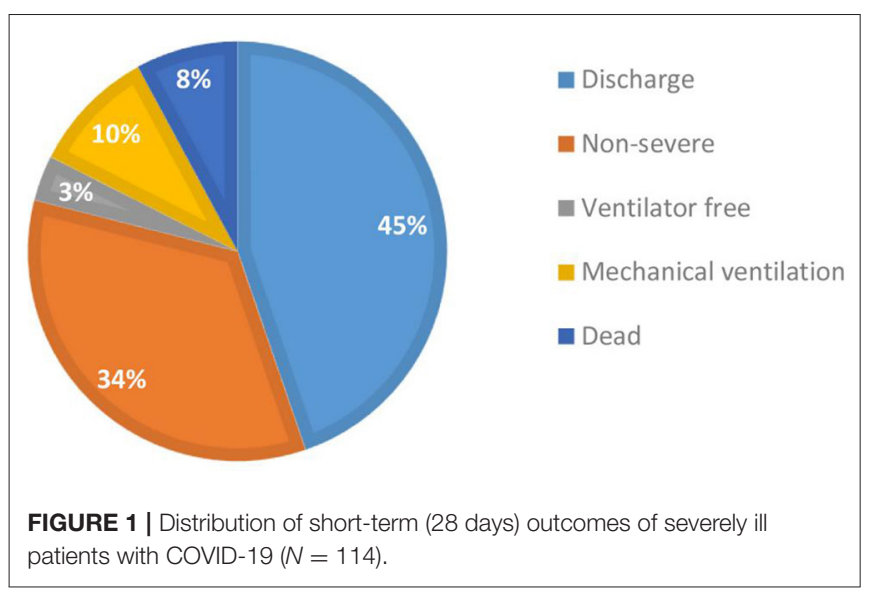

upper limit of the reference range ( $>28 \mathrm{pg} / \mathrm{mL})$, measured in the laboratory of Union Hospital (5).

\section{Statistics}

Continuous variables were expressed as means \pm SDs if normally distributed and medians (IQRs) if skewed distributed while categorical variables were summarized as number (\%). Differences between the characteristics of outcome groups were assessed using students $t$-test or Mann-Whitney U-test for continuous variables and chi-square tests for categorical variables. In addition, univariate and multivariate Cox proportional hazard models were used to determine hazard ratios (HRs) and 95\% confidence intervals (CIs) of poor outcome in severe patients with COVID-19. The candidate risk factors included demographic and epidemiological characteristics as well as some laboratory indices. We determined the cut points of levels according to normal range, actual distribution, and clinical significance of each index. Adjustments were made for potential confounders, including age and sex. For risk factors identified in Cox analyses, we used restricted cubic spline model to further explore the potential dose-response relationship between factors and poor outcome risk. The referent $(\mathrm{HR}=1)$ was set according to the cut point in Cox analyses. $P<0.05$ was considered statistically significant. All data were analyzed using SPSS (23.0 IBM SPSS).

\section{RESULTS}

\section{Clinical Outcomes}

As of March 21, 2020, a total of 114 patients diagnosed with and treated for severe COVID-19 were enrolled in this study. Twentyeight days after a diagnosis of severe COVID-19, good and poor outcomes were observed in 94 and 20 patients, respectively. As shown in Figure 1, 51 (45\%) patients were alive and had been discharged, 39 (34\%) had transitioned to non-severe illness, four (3\%) remained severely ill but did not require a ventilator, i.e., severe status and ventilator free, 11 (10\%) were alive but remained ventilated, and nine $(8 \%)$ had died.

\section{Demographics and Characteristics}

The general demographic and epidemiological characteristics of all enrolled patients are summarized in Table 1. The mean age was $63.96 \pm 13.41$ years, $62(54.4 \%)$ were older than 65 years and $71(62.3 \%)$ were male. A total of $89(78.1 \%)$ severe patients have a chronic medical illness, and the most coexisting disorders were hypertension with 62 (54.4\%), diabetes with $39(34.2 \%)$ and cardiovascular disease with 31 (27.2\%). No significant differences were observed in such characteristics between outcome groups ( $P \geq 0.05$ for all). Table 2 displays the clinical characteristics of the patients. The duration from onset of symptoms to diagnosis of COVID-19 was 4.0 (2.0-7.0) days, while the duration from the onset of symptoms to the diagnosis of severe illness was 10.0 (6.0-14.3) days. For 114 patients, the most common symptoms at initial diagnosis were fever in 78 (68.4\%), cough in $49(43.0 \%)$, chest tightness in $34(29.8 \%)$, and fatigue in $30(26.3 \%)$ patients. Other symptoms, including shortness of breath in $18(15.8 \%)$, anorexia in $12(10.5 \%)$, chill in $12(10.5 \%)$, myalgia in $10(8.8 \%)$, sputum in nine $(7.9 \%)$, headache in eight $(7.0 \%)$, diarrhea in eight $(7.0 \%)$, chest pain in three $(2.6 \%)$, stomachache in three $(2.6 \%)$ and nausea in three $(2.6 \%)$ were relatively rare. As for oxygen saturation, patients in the poor outcome group had significantly lower levels than those in the good outcome group [median (IQR): 91 (90-93\%) in the good outcome group and 81 (74-88\%) in the bad outcome group, $P<0.001$ ].

\section{Laboratory and CT Findings}

In terms of the 114 severe patients, many laboratory indicators differed significantly between outcome groups (Table 3). Compared with the good outcome group, the absolute counts of neutrophils $6.25(4.69-9.20)$ vs. 3.48 (2.54-5.23), c-reactive protein $102.15(78.07-122.90)$ vs. 48.95 (15.08-83.98), D dimer $2.10(1.22-3.07)$ vs. $0.96(0.41-.78)$, total bilirubin 19.20 (9.25-33.05) vs. 11.05 (8.53-14.05), blood urea nitrogen 9.02 (5.26-11.30) vs. 4.11 (3.11-5.04), creatine kinase 151.50 (50.50$218.50)$ vs. 62.00 (46.75-110.50), lactate dehydrogenase 638.00 (436.00-923.00) vs. 259.50 (213.75-382.50), hypersensitive cardiac troponin I 60.70 (18.48-298.98) vs. 4.10 (1.70-10.83), ferritin $679.00 \quad(573.90-993.15)$ vs. $321.80 \quad(231.00-532.88)$, interleukin-6 (IL-6) 76.10 (19.05-192.88) vs. 21.23 (7.23-47.61), and IL-10 6.59 (4.58-11.78) vs. 4.64 (3.65-6.18) were significantly higher in poor outcome. Besides, total protein 60.40 (56.78$64.05)$ vs. 63.80 (59.33-68.50) and $\mathrm{PaO} 268.15$ (49.00-77.75) vs. 81.00 (74.75-89.00) were significantly lower in poor outcome group. For chest X-ray/CT, 107 (93.9) patients had Ground-glass opacity. These data indicated that the uncontrolled inflammation responses, infection, liver, and kidney dysfunction, and hypoxia may contribute to poor outcomes in patients with COVID-19.

\section{Complications and Treatments}

As shown in Table 4, patients with severe COVID-19 had complications including acute liver injury, ARDS, acute kidney injury, arrhythmia, acute myocardial injury, Disseminated Intravascular Coagulation (DIC), rhabdomyolysis, and septic shock. However, no patients in the good outcome group experienced septic shock. All patients in the poor outcome group experienced ARDS. Acute myocardial injury, acute kidney 
TABLE 1 | Demographic and epidemiological characteristics of severe patients with COVID-19.

\begin{tabular}{|c|c|c|c|c|}
\hline & Total $(N=114)$ & Good outcome $(N=94)$ & Poor outcome $(N=20)$ & $P$ \\
\hline Age (years) & $63.96 \pm 13.41$ & $62.85 \pm 13.65$ & $69.15 \pm 11.08$ & 0.056 \\
\hline$<65$ & $52(45.6)$ & $46(48.9)$ & $6(30.0)$ & 0.123 \\
\hline$\geq 65$ & $62(54.4)$ & $48(51.1)$ & $14(70.0)$ & \\
\hline Sex, male & $71(62.3)$ & $58(61.7)$ & $13(65.0)$ & 0.782 \\
\hline Hospital infection & $7(6.1)$ & $6(6.4)$ & $1(5.0)$ & $>0.999$ \\
\hline Coexisting disorders & $89(78.1)$ & $73(77.7)$ & $16(80.0)$ & $>0.999$ \\
\hline Diabetes & $39(34.2)$ & $34(36.2)$ & $5(25.0)$ & 0.339 \\
\hline Hypertension & $62(54.4)$ & $50(53.2)$ & $12(60.0)$ & 0.579 \\
\hline Hyperlipidemia & $17(14.9)$ & $15(16.0)$ & $2(10.0)$ & 0.739 \\
\hline Cardiovascular diseases & $31(27.2)$ & $24(25.5)$ & 7 (35.0) & 0.388 \\
\hline Cerebrovascular diseases & $6(5.3)$ & $3(3.2)$ & $3(15.0)$ & 0.110 \\
\hline Cancer & $10(8.8)$ & $9(9.6)$ & $1(5.0)$ & 0.825 \\
\hline Chronic renal diseases & $6(5.3)$ & 4 (4.3) & $2(10.0)$ & 0.622 \\
\hline Chronic liver diseases & $4(3.5)$ & $3(3.2)$ & $1(5.0)$ & 0.543 \\
\hline Chronic Obstructive Pulmonary Disease & $11(9.6)$ & $9(9.6)$ & $2(10.0)$ & $>0.999$ \\
\hline Neuropsychiatric disorders & $3(2.6)$ & $2(2.1)$ & $1(5.0)$ & 0.443 \\
\hline History of surgery & $33(28.9)$ & $26(27.7)$ & $7(35.0)$ & 0.511 \\
\hline
\end{tabular}

Values are $n(\%)$ for categorical data, means $\pm S D$ s for normally distributed data, or medians (IQRs) for non-normally distributed data.

injury, arrhythmia, rhabdomyolysis, and DIC were significantly higher than their counterparts in 13.8, 22.3, 17.0, 2.1, and $2.1 \%$ of patients with COVID-19 in the good outcome group, respectively. All 114 patients with severe COVID-19 were treated with antibiotics and high flow nasal cannula, while 25 (21.9\%) were treated with non-invasive mechanical ventilation and 22 (19.3\%) with invasive mechanical ventilation treatment. Six (5.3\%) patients were treated with extracorporeal membrane oxygenation (ECMO) and all of whom were in the poor outcome group. Almost all [113 (99.1\%)] patients received antiviral treatment, including arbidol hydrochloride capsules $(0.2 \mathrm{~g}$ three times daily), lopinavir, and ribavirin (500 $\mathrm{mg}$ two times daily) via the oral route. Furthermore, as many as $41.2 \%$ patients received glucocorticoid therapy. Sixty-four (56.1\%) patients received immunoglobulin treatment, and $49(43.0 \%)$ patients were treated with parenteral nutrition; the percentage was higher in the poor outcome group than in the good outcome group [20 (100.0\%) vs. $29(30.9 \%)$ ]. Two patients $(1.8 \%)$ were treated with renal replacement therapy and $20(17.5 \%)$ with vasoconstrictive agents, and it was higher than in the good outcome group [19 (95.0\%) vs. $1(1.1 \%)]$. Moreover, the patients were given Traditional Chinese medicine (TCM) based on the protocol (9). All 20 patients in the poor group were transferred to the ICU, which was significantly higher than that of $9(9.6 \%)$ in the good outcome group.

\section{Prediction of Risk Factors for Severe COVID-19 in the Poor Outcome Group}

Tables 5, 6 display the results of univariate and multivariate Cox analyses of potential risk factors for short-term outcomes in severe patients with COVID-19. Our results indicated that, for severe patients, higher levels of oxygen saturation (HR, 0.123; 95\% CI, 0.041-0.369), albumin (HR, 0.060; 95\% CI, $0.008-0.460)$, and arterial partial pressure of oxygen (HR,
$0.321 ; 95 \%$ CI, 0.106-0.973) were associated with decreased risk of developing poor outcome within 28 days. In the other hand, higher levels of leucocytes (HR, 5.575; 95\% CI, 2.08014.943), neutrophils (HR, 2.566; 95\% CI, 1.022-6.443), total bilirubin (HR, 6.171; 95\% CI, 2.458-15.496), globulin (HR, 2.526; 95\% CI, 1.027-6.211), blood urea nitrogen (HR, 5.640; 95\% CI, 2.193-14.509), creatine kinase-MB (HR, 3.032; 95\% CI, 1.203-7.644), lactate dehydrogenase (HR, 4.607; 95\% CI, 1.057-20.090), hypersensitive cardiac troponin I (HR, 5.023; 95\% CI, 1.921-13.136), lactate concentration (HR,15.721; 95\% CI, 2.099-117.777), Interleukin-10 (HR, 3.551; 95\% CI, 1.280-9.857), and C-reactive protein ( $\mathrm{HR}, 5.275 ; 95 \% \mathrm{CI}, 1.517-18.344)$ were associated with increased risk of poor outcome development. For all the factors analyzed above, increased concentration of lactate $(\geq 1.6 \mathrm{mmol} / \mathrm{L})$ and total bilirubin $(\geq 19.0 \mu \mathrm{mol} / \mathrm{L})$ might be the most important predictors of poor outcome in the early stage. As shown in Figure 2, non-linear dose-response relationship was also found between 10 indices and poor outcome risk in the cubic spline model.

\section{DISCUSSION}

The global spread of SARS-CoV-2 poses a significant threat to public health. Previous studies have shown that $20 \%$ of COVID-19 patients developed critical disease due to hypoxia or respiratory failure. Among them, 5\% require treatment in the ICU, while $15 \%$ require oxygen and essential care. This suggests that this is particularly important in understanding this part of the patient (10). Recently, Dong et al. found that children, particularly infants, developed severe outcomes (11). This indicated that patients of any age could develop severe illness. Feng et al. found that severe and critical patients with the typical characteristics of multiple organ and immune function 
TABLE 2 | Clinical characteristics of severe patients with COVID-19.

\begin{tabular}{|c|c|c|c|c|}
\hline & $\begin{array}{c}\text { Total } \\
(N=114)\end{array}$ & $\begin{array}{l}\text { Good outcome } \\
\qquad(N=94)\end{array}$ & $\begin{array}{l}\text { Poor outcome } \\
\qquad(N=20)\end{array}$ & $P$ \\
\hline \multicolumn{5}{|l|}{ Onset of symptom to, $d$} \\
\hline Diagnosis & $4.0(2.0-7.0)$ & $4.0(2.0-7.0)$ & $4.5(2.3-10.8)$ & 0.517 \\
\hline Serious illness & $10.0(6.0-14.3)$ & $10.0(6.0-15.0)$ & $8.0(5.0-14.0)$ & 0.540 \\
\hline \multicolumn{5}{|l|}{ Signs and symptoms at initial } \\
\hline Fever & $78(68.4)$ & $63(67.0)$ & $15(75.0)$ & 0.486 \\
\hline Chest tightness & $34(29.8)$ & $27(28.7)$ & $7(35.0)$ & 0.577 \\
\hline Shortness of breath & $18(15.8)$ & $13(13.8)$ & $5(25.0)$ & 0.365 \\
\hline Cough & $49(43.0)$ & $39(41.5)$ & $10(50.0)$ & 0.485 \\
\hline Sputum & $9(7.9)$ & $8(8.5)$ & $1(5.0)$ & 0.943 \\
\hline Fatigue & $30(26.3)$ & $28(29.8)$ & $2(10.0)$ & 0.068 \\
\hline Headache & $8(7.0)$ & $7(7.4)$ & $1(5.0)$ & $>0.999$ \\
\hline Myalgia & $10(8.8)$ & $8(8.5)$ & $2(10.0)$ & $>0.999$ \\
\hline Chest pain & $3(2.6)$ & $3(3.2)$ & $0(0.0)$ & 1.000 \\
\hline Anorexia & $12(10.5)$ & $12(12.8)$ & $0(0.0)$ & 0.198 \\
\hline Chill & $12(10.5)$ & $8(8.5)$ & $4(20.0)$ & 0.263 \\
\hline Stomachache & $3(2.6)$ & $3(3.2)$ & $0(0.0)$ & $>0.999$ \\
\hline Diarrhea & $8(7.0)$ & $7(7.4)$ & $1(5.0)$ & $>0.999$ \\
\hline Nausea & $3(2.6)$ & $2(2.1)$ & $1(5.0)$ & 0.443 \\
\hline Temperature at disease onset $\left({ }^{\circ} \mathrm{C}\right)$ & $38.1(36.7-38.8)$ & $38.1(36.7-38.7)$ & $38.2(37.0-39.0)$ & 0.561 \\
\hline$<37.4$ & $36(31.6)$ & $31(33.0)$ & $5(25.0)$ & 0.622 \\
\hline $37.4-39.0$ & $63(55.3)$ & $50(53.2)$ & $13(65.0)$ & \\
\hline$>39.0$ & $15(13.2)$ & $13(13.8)$ & $2(10.0)$ & \\
\hline \multicolumn{5}{|c|}{ Signs and symptoms at hospital admission } \\
\hline Fever & $32(28.1)$ & $25(26.6)$ & $7(35.0)$ & 0.448 \\
\hline Chest tightness & $50(43.9)$ & $46(48.9)$ & $4(20.0)$ & 0.018 \\
\hline Shortness of breath & $68(59.6)$ & $49(52.1)$ & $19(95.0)$ & $<0.001$ \\
\hline Cough & $9(7.9)$ & $7(7.4)$ & $2(10.0)$ & $>0.999$ \\
\hline Fatigue & $43(37.7)$ & $37(39.4)$ & $6(30.0)$ & 0.433 \\
\hline Headache & $6(5.3)$ & $6(6.4)$ & $0(0.0)$ & 0.542 \\
\hline Myalgia & $5(4.4)$ & $5(5.3)$ & $0(0.0)$ & 0.585 \\
\hline Chest pain & $3(2.6)$ & $3(3.2)$ & $0(0.0)$ & $>0.999$ \\
\hline Anorexia & $18(15.8)$ & $16(17.0)$ & $2(10.0)$ & 0.657 \\
\hline Diarrhea & $10(8.8)$ & $7(7.4)$ & $3(15.0)$ & 0.516 \\
\hline Nausea & $2(1.8)$ & $2(2.1)$ & $0(0.0)$ & $>0.999$ \\
\hline \multicolumn{5}{|l|}{ General signs at admission } \\
\hline Temperature $\left({ }^{\circ} \mathrm{C}\right)$ & $36.7(36.4-37.4)$ & $36.7(36.4-37.3)$ & $38.2(37.0-39.0)$ & 0.279 \\
\hline$<37.4$ & $85(74.6)$ & $72(76.6)$ & $13(65.0)$ & 0.535 \\
\hline $37.4-39.0$ & $26(22.8)$ & $20(21.3)$ & $6(30.0)$ & \\
\hline$>39.0$ & $3(2.6)$ & $2(2.1)$ & $1(5.0)$ & \\
\hline Heart rate (/min) & $86(78-102)$ & $87(78-102)$ & $88(78-102)$ & 0.456 \\
\hline$<100$ & $83(72.8)$ & $69(73.4)$ & $14(70.0)$ & 0.756 \\
\hline$>100$ & $31(27.2)$ & 25 (26.6) & $6(30.0)$ & \\
\hline Respiratory rate (/min) & $20(20-23)$ & $20(20-22)$ & $21(20-30)$ & 0.122 \\
\hline$<20$ & 22 (19.3) & $19(20.2)$ & $3(15.0)$ & 0.760 \\
\hline$\geq 20$ & $92(80.7)$ & 75 (79.8) & 17 (85.0) & \\
\hline Oxygen saturation (\%) & $90(88-92)$ & $91(90-93)$ & $81(74-88)$ & $<0.001$ \\
\hline$<90$ & $35(30.7)$ & $19(20.2)$ & $16(80.0)$ & $<0.001$ \\
\hline$\geq 90$ & $79(69.3)$ & 75 (79.8) & $4(20.0)$ & \\
\hline
\end{tabular}

Values are $n(\%)$ for categorical data, means \pm SDs for normally distributed data, or medians (IQRs) for non-normally distributed data. 
TABLE 3 | Laboratory and radiographic findings at baseline of severe patients with COVID-19.

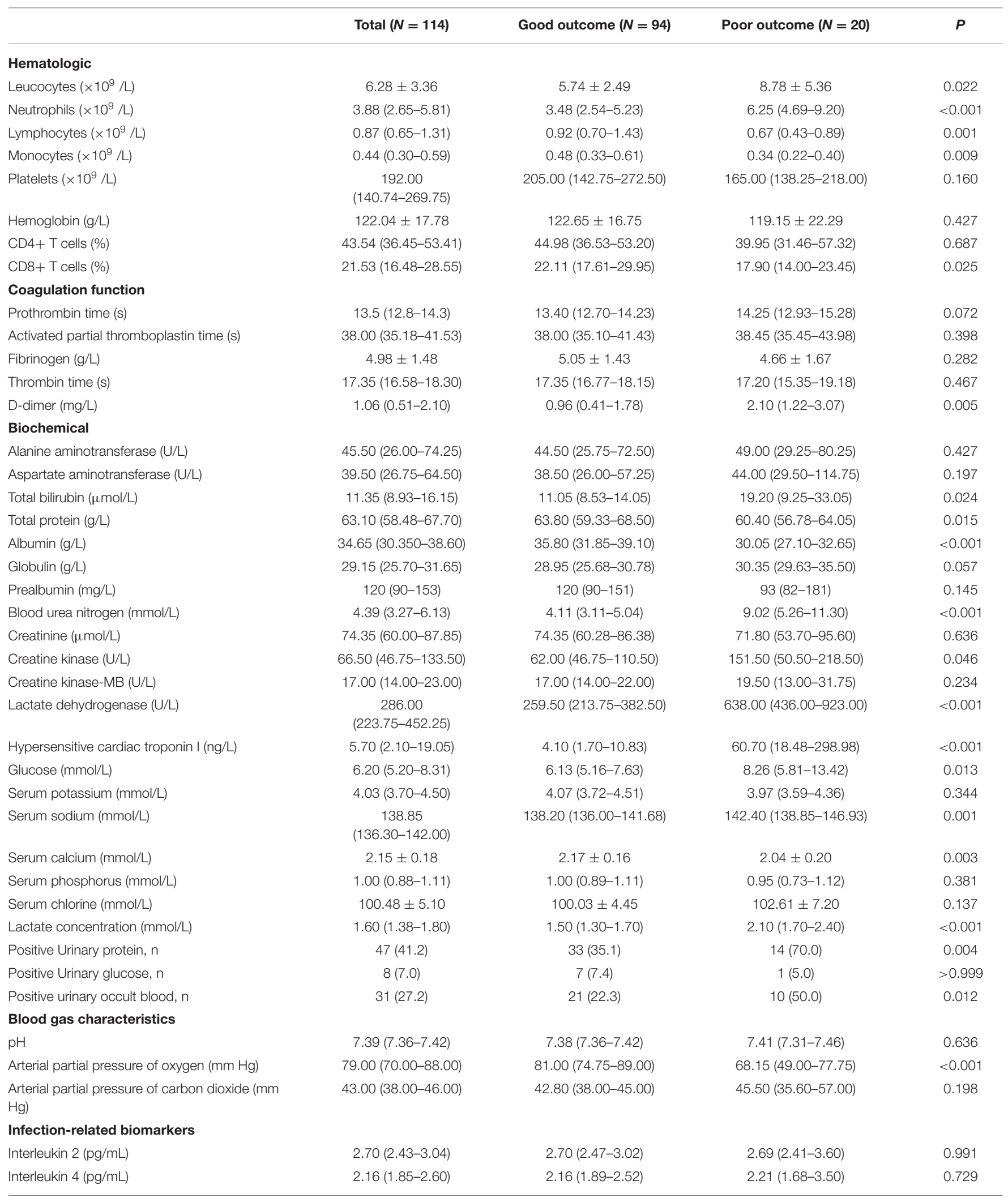


TABLE 3 | Continued

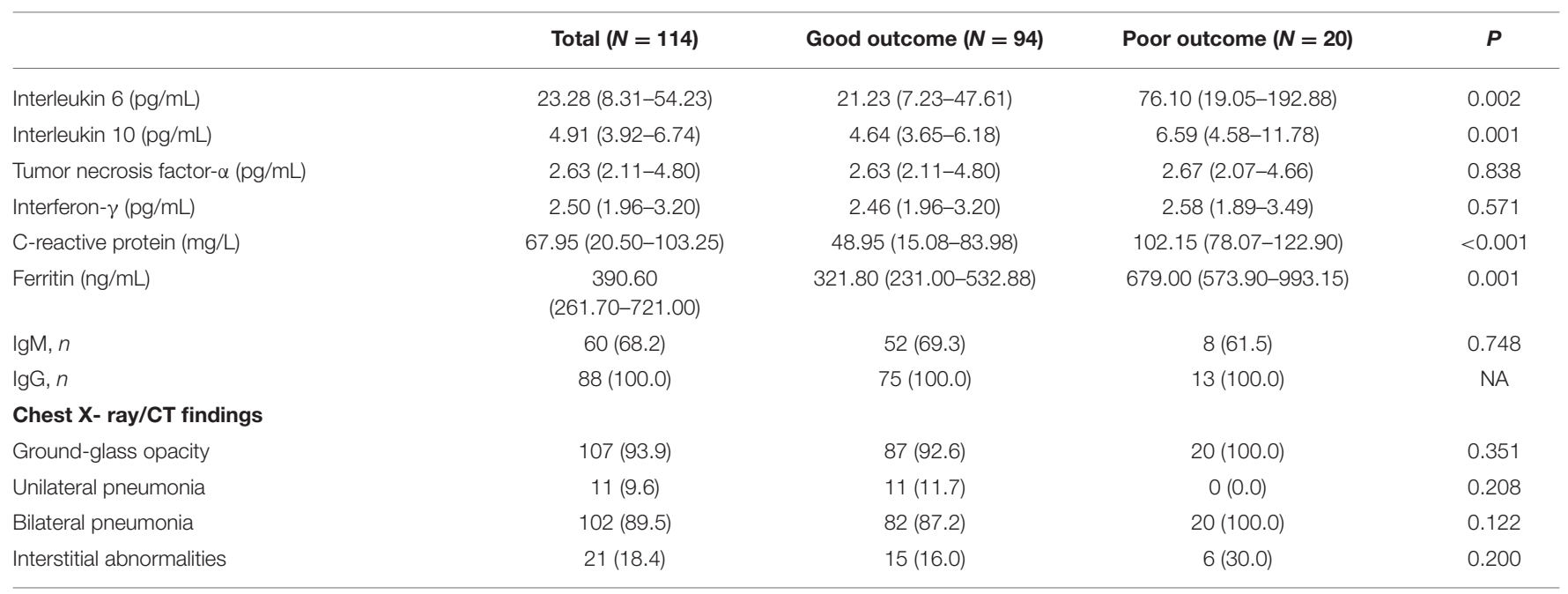

Data are $n(\%)$, means \pm SDs or medians (IQRS) when appropriate.

TABLE 4 | Complications and treatments of severe patients with COVID-19.

\begin{tabular}{|c|c|c|c|c|}
\hline & $\begin{array}{c}\text { Total } \\
(N=114)\end{array}$ & $\begin{array}{l}\text { Good outcome } \\
\qquad(N=94)\end{array}$ & $\begin{array}{l}\text { Poor outcome } \\
\qquad(N=20)\end{array}$ & $P$ \\
\hline \multicolumn{5}{|l|}{ Complications } \\
\hline Acute respiratory distress syndrome & $41(36.0)$ & $21(22.3)$ & $20(100.0)$ & $<0.001$ \\
\hline Acute renal injury & $35(30.7)$ & $21(22.3)$ & $14(70.0)$ & $<0.001$ \\
\hline Arrhythmia & $31(27.2)$ & $16(17.0)$ & $15(75.0)$ & $<0.001$ \\
\hline Rhabdomyolysis & $11(9.6)$ & $2(2.1)$ & $9(45.0)$ & $<0.001$ \\
\hline Disseminated intravascular coagulation & $15(13.2)$ & $2(2.1)$ & $13(65.0)$ & $<0.001$ \\
\hline \multicolumn{5}{|l|}{ Treatment } \\
\hline Antibiotic treatment & $114(100.0)$ & $94(100.0)$ & $20(100.0)$ & NA \\
\hline Immunoglobulin & $64(56.1)$ & $45(47.9)$ & $19(95.0)$ & $<0.001$ \\
\hline Parenteral nutrition & $49(43.0)$ & $29(30.9)$ & 20 (100.0) & $<0.001$ \\
\hline Admission to intensive care unit & $29(25.4)$ & $9(9.6)$ & $20(100.0)$ & $<0.001$ \\
\hline Non-invasive ventilation & $25(21.9)$ & $13(13.8)$ & $12(60.0)$ & $<0.001$ \\
\hline Invasive mechanical ventilation & 22 (19.3) & $4(4.3)$ & $18(90.0)$ & $<0.001$ \\
\hline Extracorporeal membrane oxygenation & $6(5.3)$ & $0(0.0)$ & $6(30.0)$ & $<0.001$ \\
\hline Vasoconstrictive agents & $20(17.5)$ & $1(1.1)$ & $19(95.0)$ & $<0.001$ \\
\hline Renal replacement therapy & $2(1.8)$ & $0(0.0)$ & $2(10.0)$ & 0.029 \\
\hline Traditional Chinese medicine & $86(75.4)$ & 77 (81.9) & $9(45.0)$ & 0.001 \\
\hline Trastuzumab & $13(11.4)$ & $12(12.8)$ & $1(5.0)$ & 0.459 \\
\hline Infusions of blood plasma & $4(3.5)$ & $2(2.1)$ & $2(10.0)$ & 0.141 \\
\hline
\end{tabular}

Data are $n$ (\%) or medians (IQRs) when appropriate.

*Data available for 98 patients. 
TABLE 5 | Univariate and multivariate analyses of potential factors (demographic and epidemiologic) predicting poor outcome.

\begin{tabular}{|c|c|c|c|c|c|}
\hline Factors & Level & Crude HR (95\% Cl) & $P$ & Adjusted HR $(95 \% \mathrm{CI})^{\star}$ & $\boldsymbol{P}$ \\
\hline Age (years) & $\geq 65$ vs. $<65$ & $2.192(0.842-5.708)$ & 0.108 & $2.184(0.839-5.687)$ & 0.110 \\
\hline Sex & Female vs. Male & $0.772(0.308-1.937)$ & 0.581 & $0.732(0.292-1.838)$ & 0.507 \\
\hline Coexisting disorders & Yes vs. No & $1.154(0.386-3.453)$ & 0.797 & $0.692(0.207-2.313)$ & 0.550 \\
\hline Diabetes & Yes vs. No & $0.706(0.257-1.945)$ & 0.380 & $0.622(0.224-1.728)$ & 0.363 \\
\hline Hypertension & Yes vs. No & $1.122(0.458-2.747)$ & 0.801 & $0.960(0.386-2.384)$ & 0.929 \\
\hline Hyperlipidemia & Yes vs. No & $0.677(0.157-2.919)$ & 0.601 & $0.729(0.168-3.167)$ & 0.673 \\
\hline Cardiovascular diseases & Yes vs. No & $1.601(0.638-4.015)$ & 0.316 & 1.062 (0.380-2.970) & 0.908 \\
\hline Cerebrovascular diseases & Yes vs. No & $3.327(0.975-11.356)$ & 0.055 & $2.326(0.612-8.848)$ & 0.216 \\
\hline Cancer & Yes vs. No & $0.536(0.072-4.004)$ & 0.543 & $0.410(0.054-3.103)$ & 0.388 \\
\hline Chronic renal diseases & Yes vs. No & $3.678(0.835-16.202)$ & 0.085 & 3.437 (0.764-15.465) & 0.108 \\
\hline Chronic liver diseases & Yes vs. No & $1.433(0.192-10.707)$ & 0.726 & $0.997(0.128-7.760)$ & 0.997 \\
\hline Chronic Obstructive Pulmonary Disease & Yes vs. No & $0.991(0.230-4.272)$ & 0.990 & $0.642(0.139-2.955)$ & 0.569 \\
\hline Neuropsychiatric disorders & Yes vs. No & $1.186(0.158-8.894)$ & 0.868 & $0.734(0.094-5.741)$ & 0.768 \\
\hline History of surgery & Yes vs. No & $1.168(0.466-2.930)$ & 0.740 & $1.041(0.413-2.623)$ & 0.932 \\
\hline \multirow[t]{2}{*}{ Temperature at disease onset $\left({ }^{\circ} \mathrm{C}\right)$} & $37.4-39.0$ vs. $<37.4$ & $1.514(0.540-4.248)$ & 0.430 & $1.844(0.638-5.326)$ & 0.258 \\
\hline & $>39.0$ vs. $<37.4$ & $1.036(0.201-5.342)$ & 0.966 & $2.714(0.348-21.190)$ & 0.622 \\
\hline \multirow[t]{2}{*}{ Temperature at admission $\left({ }^{\circ} \mathrm{C}\right)$} & $37.4-39.0$ vs. $<37.4$ & $1.476(0.561-3.885)$ & 0.430 & $1.721(0.646-4.580)$ & 0.277 \\
\hline & $>39.0$ vs. $<37.4$ & $2.630(0.343-20.167)$ & 0.352 & $2.714(0.348-21.190)$ & 0.341 \\
\hline Respiratory rate (/min) & $\geq 20$ vs. $<20$ & 1.357 (0.398-4.629) & 0.626 & $1.316(0.385-4.502)$ & 0.662 \\
\hline Oxygen saturation (\%) & $\geq 90$ vs. $<90$ & $0.131(0.044-0.394)$ & $<0.001$ & $0.123(0.041-0.369)$ & $<0.001$ \\
\hline
\end{tabular}

*Adjustments were made for age and sex.

dysfunction. They also found that older people aged $\geq 75$ years are a risk factor for mortality (12). With the increase number of asymptomatic infectious patients, taking measures to detect and isolate early are especially important. In our study, we used a short-term method to prospectively study the reported the epidemiology and risk factors of 114 severe patients with COVID-19 from the Union hospital, Hubei province. To our knowledge, this is the first report to describes the severe patients with COVID-19 during a short-term observation and predict some risk factors for final outcome. In our study, the mean age of severe patients were $63.96 \pm 13.41$ years, and 58 (50.9\%) were older than 65 years; the patients are thus older than in other studies $(5,13,14)$. We also found that $78(68.4 \%)$ of 114 patients initially exhibited fever, in accordance with previous studies, where fever is the one of the most common symptom in patients who had COVID-19 (5, 14-16). But, 36 (31.6\%) of 114 severe patients did not exhibit fever at the beginning of illness, and other clinical manifestations should therefore be concerned. Recently, Jin et al. found that attention should also be paid to people who have gastrointestinal symptoms (6). Mao et al. indicated that clinicians should suspect COVID-19 also in patients with neurological manifestations (14).

According to results from laboratory tests, the poor outcome group had lower lymphocytes than the good outcome group [0.67 (0.43-0.89) vs. $0.92(0.70-1.43)]$. As is known to all, lymphocytes are the main fighting force against the virus, and we suspected that SARS-CoV-2 damages the lymphocyte and causes its reduction (17). Chen et al. found that severe lymphopenia were persistent and we more increased in dead patients than recovered patients, and they suggested that lymphopenia may be associated with poor outcome (18). Tan et al. demonstrated a contrasting result: lymphopenia is an effective indicator for the severity of patients with COVID-19 (19). CD8+ T cells were significantly lower in the poor outcome group. Chen et al. indicated that the SARS-CoV-2 infection may affect CD4+ and CD8 + T lymphocyte cells in particular and argue that this is a potential correlation with COVID-19 severity (20). In addition, markedly higher concentrations of cardiac troponin I, creatine kinase, and lactate dehydrogenase could be observed in the poor outcome group than in their counterpart. Most notably, patients who exhibited a poor outcome may develop pulmonary and extra-pulmonary organ damage, including septic shock, acute respiratory distress syndrome, acute kidney injury, acute cardiac injury, as well as disseminated intravascular coagulation. The fatality risk of COVID-19 patients with or without a history of previous cardiovascular disease may include acute cardiac injury and heart failure (18). Costanza Emanueli et al. suggested that the COVID-19 crisis will have long-term residual repercussions on the cardiovascular system (21). The suggestion is that the cardiac injury also requires special attention. In our study, we also found that lactate concentration was higher in poor outcomes than their counterpart. Lactate is generally the end product of energy through anaerobic metabolism, and the elevation of lactate levels is mainly caused by the increase of blood oxygen deficiency and anaerobic metabolism; this result is consistent with the lower oxygen saturation in the poor group, and this indicated that lactate level is an important predictor of poor outcome in the early stage. In addition, total bilirubin was also an important predictor of poor outcome in the early stage. Qi recommend that dynamic monitoring of the liver function of 
TABLE 6 | Univariate and multivariate analyses of potential factors (laboratory indexes) predicting poor outcome.

\begin{tabular}{|c|c|c|c|c|c|c|}
\hline Factors & Normal range & Level $^{\dagger}$ & Crude HR (95\% Cl) & $P$ & Adjusted HR $(95 \% \mathrm{Cl})^{*}$ & $P$ \\
\hline \multicolumn{7}{|l|}{ Hematologic } \\
\hline Leucocytes (×109 /L) & $3.5-9.5$ & $\geq 9.5$ vs. $<9.5$ & $4.634(1.840-11.669)$ & 0.001 & $5.575(2.080-14.943)$ & 0.001 \\
\hline Lymphocytes $\left(\times 10^{9} / L\right)$ & $1.1-3.2$ & $\geq 1.1$ vs. $<1.1$ & $0.293(0.068-1.266)$ & 0.100 & $0.337(0.077-1.475)$ & 0.149 \\
\hline Monocytes (×109/L) & $0.1-0.6$ & $\geq 0.6$ vs. $<0.6$ & $0.179(0.024-1.335)$ & 0.179 & $0.182(0.024-1.366)$ & 0.098 \\
\hline Platelets $\left(\times 10^{9} / \mathrm{L}\right)$ & $125.0-350.0$ & $\geq 125.0$ vs. $<125.0$ & $0.733(0.245-2.192)$ & 0.578 & $0.837(0.275-2.553)$ & 0.755 \\
\hline CD8+ T cells (\%) & $14.23-38.95$ & $\geq 14.23$ vs. $<14.23$ & $0.687(0.249-1.890)$ & 0.467 & $0.867(0.303-2.482)$ & 0.790 \\
\hline \multicolumn{7}{|l|}{ Coagulation function } \\
\hline Prothrombin time (s) & $11.0-16.0$ & $\geq 13.5$ vs. $<13.5$ & $1.574(0.644-3.852)$ & 0.320 & $1.112(0.417-2.966)$ & 0.832 \\
\hline Activated partial thromboplastin time (s) & $28.0-43.5$ & $\geq 43.5$ vs. $<43.5$ & $1.363(0.493-3.766)$ & 0.551 & $1.204(0.433-3.344)$ & 0.722 \\
\hline Fibrinogen (g/L) & $2.0-4.0$ & $\geq 4.0$ vs. $<4.0$ & $0.533(0.212-1.338)$ & 0.180 & $0.520(0.205-1.317)$ & 0.168 \\
\hline Aspartate aminotransferase (U/L) & $8-40$ & $\geq 40$ vs. $<40$ & $1.611(0.658-3.942)$ & 0.296 & $1.900(0.755-4.783)$ & 0.173 \\
\hline Total bilirubin ( $\mu \mathrm{mol} / \mathrm{L})$ & $5.1-19.0$ & $\geq 19.0$ vs. $<19.0$ & $5.849(2.433-14.063)$ & $<0.001$ & $6.171(2.458-15.496)$ & $<0.001$ \\
\hline Total protein (g/L) & $60-80$ & $\geq 60$ vs. $<60$ & $0.687(0.284-1.661)$ & 0.405 & $0.721(0.298-1.748)$ & 0.470 \\
\hline Albumin (g/L) & $35-55$ & $\geq 35$ vs. $<35$ & $0.054(0.007-0.405)$ & 0.054 & $0.060(0.008-0.460)$ & 0.007 \\
\hline Globulin (g/L) & $20-30$ & $\geq 30$ vs. $<30$ & $2.723(1.113-6.666)$ & 0.028 & $2.526(1.027-6.211)$ & 0.043 \\
\hline Prealbumin (mg/L) & $170-420$ & $\geq 170$ vs. $<170$ & $1.001(0.364-2.755)$ & 0.999 & $1.282(0.448-3.665)$ & 0.643 \\
\hline Blood urea nitrogen (mmol/L) & $2.9-8.2$ & $\geq 8.2$ vs. $<8.2$ & $6.283(2.565-15.391)$ & $<0.001$ & $5.640(2.193-14.509)$ & $<0.001$ \\
\hline Creatinine $(\mu \mathrm{mol} / \mathrm{L})$ & $44-133$ & $\geq 74$ vs. $<74$ & $0.973(0.405-2.337)$ & 0.950 & $0.709(0.254-1.978)$ & 0.511 \\
\hline Creatine kinase (U/L) & $38-174$ & $\geq 174$ vs. $<174$ & $2.039(0.783-5.307)$ & 0.144 & $1.982(0.756-5.199)$ & 0.164 \\
\hline Creatine kinase-MB (U/L) & $0-24$ & $\geq 24$ vs. $<24$ & $2.449(1.000-5.997)$ & 0.050 & $3.032(1.203-7.644)$ & 0.019 \\
\hline Lactate dehydrogenase (U/L) & $109-245$ & $\geq 245$ vs. $<245$ & $3.963(0.915-17.161)$ & 0.066 & $4.607(1.057-20.090)$ & 0.042 \\
\hline Positive Urinary protein & / & / & $3.239(1.244-8.433)$ & 0.016 & $2.905(1.099-7.678)$ & 0.032 \\
\hline Positive Urinary glucose & / & / & $0.893(0.120-6.676)$ & 0.912 & $0.961(0.128-7.238)$ & 0.969 \\
\hline Positive urinary occult blood & / & / & $2.474(1.030-5.945)$ & 0.043 & $2.247(0.932-5.421)$ & 0.071 \\
\hline \multicolumn{7}{|l|}{ Blood gas characteristics } \\
\hline $\mathrm{pH}$ & $7.35-7.45$ & $\geq 7.35$ vs. $<7.35$ & $0.427(0.170-1.074)$ & 0.071 & $0.468(0.181-1.207)$ & 0.116 \\
\hline Arterial partial pressure of oxygen $(\mathrm{mm} \mathrm{Hg})$ & $80-100$ & $\geq 80$ vs. $<80$ & $0.295(0.098-0.883)$ & 0.029 & $0.321(0.106-0.973)$ & 0.045 \\
\hline Arterial partial pressure of carbon dioxide $(\mathrm{mm} \mathrm{Hg})$ & $35-45$ & $\geq 45$ vs. $<45$ & $2.159(0.892-5.230)$ & 0.088 & $2.224(0.895-5.525)$ & 0.085 \\
\hline \multicolumn{7}{|l|}{ Infection-related biomarkers } \\
\hline Interleukin-2 (pg/mL) & $0.1-4.1$ & $\geq 4.1$ vs. $<4.1$ & $1.820(0.533-6.212)$ & 0.339 & $2.343(0.654-8.389)$ & 0.191 \\
\hline Interleukin-4 (pg/mL) & $0.1-3.2$ & $\geq 3.2$ vs. $<3.2$ & $1.663(0.663-4.172)$ & 0.279 & $2.112(0.797-5.597)$ & 0.133 \\
\hline Interleukin-6 (pg/mL) & $0.1-2.9$ & $\geq 23.3$ vs. $<23.3$ & $1.782(0.711-4.468)$ & 0.218 & $1.485(0.575-3.836)$ & 0.414 \\
\hline Interleukin-10 (pg/mL) & $0.1-5.0$ & $\geq 5.0$ vs. $<5.0$ & $2.629(1.010-6.843)$ & 0.048 & $3.551(1.280-9.857)$ & 0.015 \\
\hline Tumor necrosis factor- $\alpha(\mathrm{pg} / \mathrm{mL})$ & $0.1-23.0$ & $\geq 2.6$ vs. $<2.6$ & $1.079(0.448-2.600)$ & 0.866 & $1.032(0.427-2.491)$ & 0.945 \\
\hline Interferon- $\gamma(\mathrm{pg} / \mathrm{mL})$ & $0.1-18.0$ & $\geq 2.5$ vs. $<2.5$ & $1.260(0.522-3.043)$ & 0.607 & $1.421(0.582-3.473)$ & 0.440 \\
\hline C-reactive protein (mg/L) & $<8.0$ & $\geq 65.0$ vs. $<65.0$ & 4.703 (1.374-16.093) & 0.014 & $5.275(1.517-18.344)$ & 0.009 \\
\hline Ferritin (ng/mL) & 4.6-204.0 & $\geq 204.0$ vs. $<204.0$ & $2.582(0.210-11.930)$ & 0.657 & 2.647 (0.311-22.522) & 0.373 \\
\hline
\end{tabular}

*Adjustments were made for age and sex.

${ }^{\dagger}$ Cut points of levels were determined according to normal range, actual distribution, and clinical significance. 
A

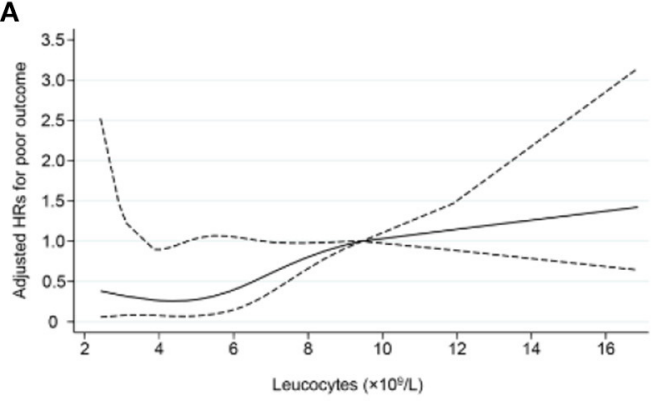

C

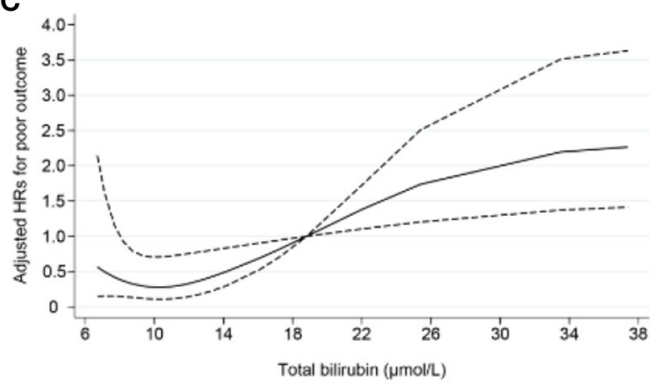

E

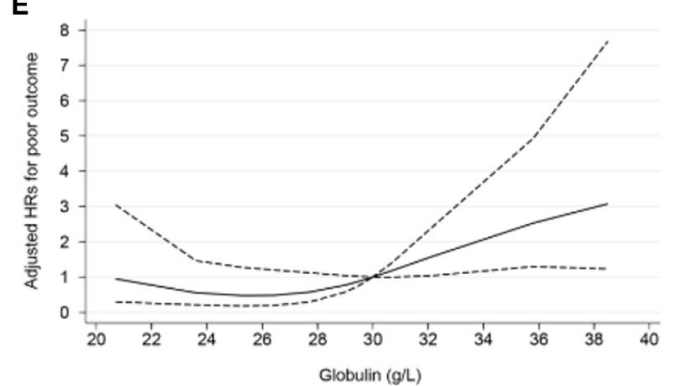

G

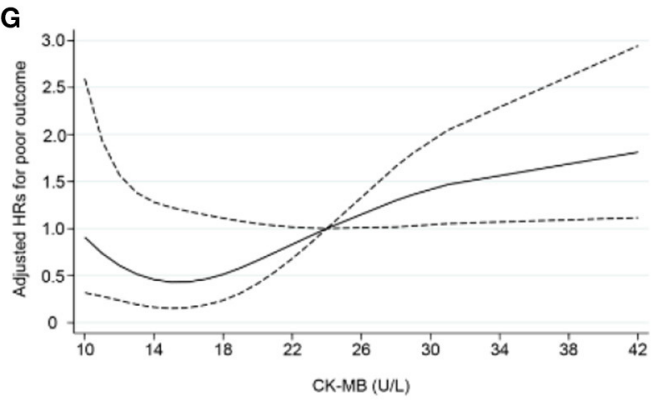

I

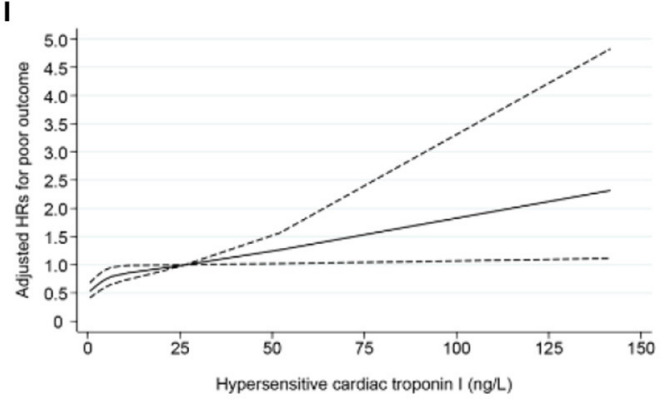

B

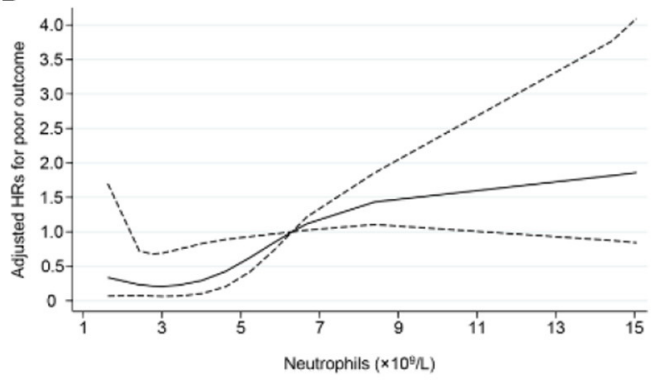

D

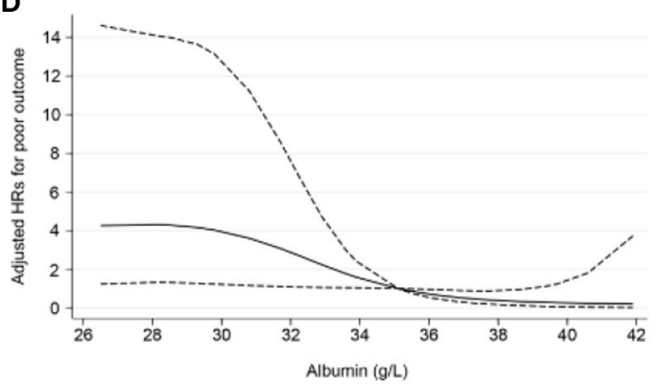

$\mathbf{F}$

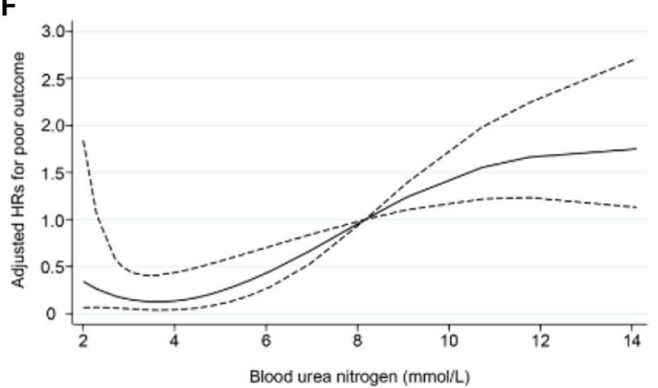

H

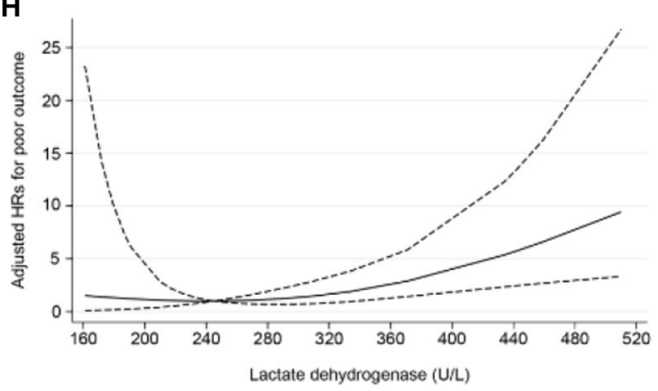

J

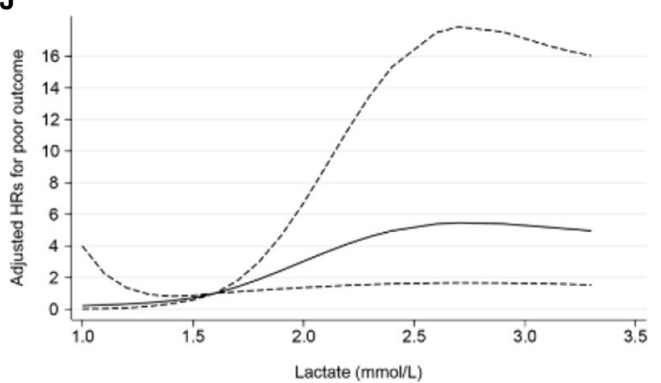

FIGURE 2 | Non-linear dose-response relationship between 10 indices and poor outcome risk. Hazard ratios (HRs) were adjusted for age and gender. Dotted lines represent the $95 \% \mathrm{Cls}$ for the fitted trend. (A) Leucocytes $\left(\times 10^{9} / \mathrm{L}\right)$, referent $(H R=1): 9.5$; (B) Neutrophils $\left(\times 10^{9} / \mathrm{L}\right)$, referent: 6.3; (C) Total bilirubin ( $\mu$ mol/L), referent: 19.0; (D) Albumin, referent: 35.0 (g/L); (E) Globulin (g/L), referent: 30.0; (F) Blood urea nitrogen (mmol/L), referent: 8.2; (G) CK-MB (U/L), referent: 24.0; (H) Lactate dehydrogenase (U/L), referent: 245; (I) Hypersensitive cardiac troponin I (ng/L), referent: 26.2; (J) Lactate (mmol/L), referent: 1.6. 
patients is necessary (22). Cai et al. conclude that patients with abnormal liver function may had higher risks of progressing to severe disease (23). Due to the "cytokine storm" also observed in the poor outcome group, 19 (95.0\%) of these patients were given glucocorticoid therapy. Wu et al. previously found that the administration of methylprednisolone may have reduced the risk of death in patients with ARDS (7). To our surprise, most of the severe patients treated with Traditional Chinese medicine (TCM) were eventually converted to a good outcome, indicating the importance of this effort on COVID-19. A large of clinical practice results indicated that TCM shows significant role in the patients with COVID-19. For the severe patients in the treatment of TCM, the mean length in hospital and the time of nucleic acid turning negative has been shortened by more than 2 days (24). Yang et al. analyzed the effect of Lian Hua Qing Wen Capsules in the treatment of COVID-19 patients, and they found that this TCM could markedly relieve fever and cough and promote recovery (25). Besides, a comprehensive evaluation and further scientific research should be carried out on the effect of TCM on COVID-19.

Meantime, the risk factors related to the poor outcome included uncontrolled inflammation responses, infection, hypoxia, and liver, kidney, and cardiac dysfunction. The pathogenesis of COVID-19 is still being studied. Cytokine storms and uncontrolled inflammation responses are thought to play important roles in the outcome of COVID-19 (26-30). External stimuli resulted in an excessive immune response, and the pathogenesis of the cytokine storm is complex and can leaded to rapid disease progression and high mortality. The inflammatory cytokine storm is closely correlated to the development and progression of ARDS (31). Neutrophils play important role in chemokines and cytokines (32). In our study, the poor outcome group had significantly higher neutrophil counts than the good outcome group, and this may be the underlying cause of the cytokine storm. In addition, $\mathrm{CD} 8^{+}$ $\mathrm{T}$ cells were significantly lower in the poor outcome group. These results highlight the important roles of $\mathrm{CD}^{+} \mathrm{T}$ cells in COVID-19. Studies had shown that $\mathrm{T}$ cells could inhibit the over-activation of innate immunity (33). T cells can help to clear SARS-CoV, and a low T-cell response can result in pathological changes in mice with SARS-CoV (34). The relevant mechanisms need to be studied further.

This study has some limitations. First, owing to the limited number of cases, only 114 severe patients were included. Second, this study was a single-center research, and a larger cohort study of severe patients with SARS-CoV-2 from other cities in China and other countries would help to further describe the clinical characteristics and predict risk factors related to

\section{REFERENCES}

1. Zhu N, Zhang D, Wang W, Li X, Yang B, Song J, et al. A novel coronavirus from patients with pneumonia in China, 2019. N Engl J Med. (2020) 382:72733. doi: 10.1056/NEJMoa2001017

2. Huang C, Wang Y, Li X, Ren L, Zhao J, Hu Y, et al. Clinical features of patients infected with 2019 novel coronavirus in Wuhan, this disease. Third, although we included numerous factors that may be associated with clinical outcome in the analyses and made adjustment for potential confounders when exploring the associations, we could not rule out the possibility of other residual confounders.

In summary, the present study is a single-center, prospective observational study that examined clinical characteristics and risk factors for poor short-term outcomes in patients with severe COVID-19. Our univariate and multivariate analyses demonstrated that cytokine storm/uncontrolled inflammatory responses as well as liver, kidney, and cardiac dysfunction may play important roles in determining final outcomes in patients with severe illness due to COVID-19 infection. Our data may aid clinicians in diagnosing severe cases of COVID19 and determining the most appropriate treatment strategies for infected patients. Given that Traditional Chinese medicine has been shown to improve outcomes in some cases, additional studies are also required to assess the efficacy of such strategies.

\section{DATA AVAILABILITY STATEMENT}

The raw data supporting the conclusions of this article will be made available by the authors, without undue reservation.

\section{ETHICS STATEMENT}

The studies involving human participants were reviewed and approved by the Ethics Commission of Wuhan Union Hospital of Tongji Medical College, Huazhong University of Science and Technology. Written informed consent was waived for the emergency of this infectious disease.

\section{AUTHOR CONTRIBUTIONS}

CY and WY designed the study, had full access to all data in the study, and took responsibility for the integrity of data and the accuracy of the data analysis. XF and LM contributed to data collection, literature search, and writing of the manuscript. XF and PL had roles in data analysis and data interpretation. All authors contributed to data acquisition and clinical management, and they reviewed and approved the final version of the manuscript.

\section{ACKNOWLEDGMENTS}

We thank all the patients and their families involved in this study as well as numerous civilians working together to fight against SARS-CoV-2. 
(SARS-Cov-2) outside of Wuhan, China: retrospective case series. BMJ. (2020) 368:m606. doi: 10.1136/bmj.m606

5. Yang X, Yu Y, Xu J, Shu H, Xia Ja, Liu H, et al. Clinical course and outcomes of critically ill patients with SARS-CoV-2 pneumonia in Wuhan, China: a single-centered, retrospective, observational study. Lancet Resp Med. (2020) 8:475-81. doi: 10.1016/S2213-2600(20)30079-5

6. Jin X, Lian JS, Hu JH, Gao J, Zheng L, Zhang YM, et al. Epidemiological, clinical and virological characteristics of 74 cases of coronavirus-infected disease (2019). (COVID-19) with gastrointestinal symptoms. Gut. (2020) 69:1002-100. doi: 10.1136/gutjnl-2020-320926

7. Wu C, Chen X, Cai Y, Xia J, Zhou X, Xu S, et al. Risk factors associated with acute respiratory distress syndrome and death in patients with Coronavirus Disease (2019). pneumonia in Wuhan, China. JAMA Intern Med. (2020) 180:1-11. doi: 10.1001/jamainternmed.2020.0994

8. Guo W, Li M, Dong Y, Zhou H, Zhang Z, Tian C, et al. Diabetes is a risk factor for the progression and prognosis of COVID-19. Diabetes Metab Res Rev. (2020) doi: 10.1002/dmrr.3319. [Epub ahead of print].

9. Released by National Health Commission National Administration of Traditional Chinese Medicine on March 3. Diagnosis and treatment protocol for novel coronavirus pneumonia (Trial Version 7). Chin Med J. (2020) 133:1087-95. doi: 10.1097/CM9.0000000000000819

10. Wu Z, McGoogan J. Characteristics of and important lessons from the Coronavirus Disease 2019. (COVID-19) outbreak in China. JAMA. (2020) 323:1239-42. doi: 10.1001/jama.2020.2648

11. Dong Y, Mo X, Hu Y, Qi X, Jiang F, Jiang Z, et al. Epidemiology of COVID-19 among children in China. Pediatrics. (2020) 145:e20200702. doi: 10.1542/peds.2020-0702

12. Feng Y, Ling Y, Bai T, Xie Y, Huang J, Li J, et al. COVID-19 with different severity_a multi-center study of clinical features. Am J Respir Crit Care Med. (2020) 201:1380-8. doi: 10.1164/rccm.202002-0445OC

13. Chen N, Zhou M, Dong X, Qu J, Gong F, Han Y, et al. Epidemiological and clinical characteristics of 99 cases of 2019 novel coronavirus pneumonia in Wuhan, China: a descriptive study. Lancet. (2020) 395:507-13. doi: 10.1016/S0140-6736(20)30211-7

14. Mao L, Jin H, Wang M, Hu Y, Chen S, He Q, et al. Neurologic manifestations of hospitalized patients with Coronavirus Disease 2019 in Wuhan, China. JAMA Neurol. (2020) 77:1-9. doi: 10.1001/jamaneurol.2020.1127

15. Wang D, Hu B, Hu C, Zhu F, Liu X, Zhang J, et al. Clinical characteristics of 138 hospitalized patients with 2019 novel coronavirus-infected pneumonia in Wuhan, China. JAMA. (2020) 323:1061-69. doi: 10.1001/jama.2020.1585

16. Wang Z, Chen X, Lu Y, Chen F, Zhang W. Clinical characteristics and therapeutic procedure for four cases with 2019 novel coronavirus pneumonia receiving combined Chinese and Western medicine treatment. Biosci Trends. (2020) 14:64-8. doi: 10.5582/bst.2020.01030

17. Gu J, Gong E, Zhang B, Zheng J, Gao Z, Zhong Y, et al. Multiple organ infection the pathogenesis of SARS. J Exp Med. (2005) 202:41524. doi: $10.1084 /$ jem. 20050828

18. Chen T, Wu D, Chen H, Yan W, Yang D, Chen G, et al. Clinical characteristics of 113 deceased patients with coronavirus disease 2019: retrospective study. BMJ. (2020) 368:m1091. doi: 10.1136/bmj.m1091

19. Tan L, Wang Q, Zhang D, Ding J, Huang Q, Tang YQ, et al. Lymphopenia predicts disease severity of COVID-19: a descriptive and predictive study. Signal Transduct Target Ther. (2020) 5:33. doi: 10.1038/s41392-020-0148-4

20. Chen G, Wu D, Guo W, Cao Y, Huang D, Wang H, et al. Clinical and immunological features of severe and moderate coronavirus disease 2019. J Clin Invest. (2020) 130:2620-9. doi: 10.1101/2020.02.16.20023903
21. Emanueli C, Badimon L, Martelli F, Potocnjak I, Carpusca I, Robinson EL, et al. Call to action for the cardiovascular side of COVID-19. Eur Heart J. (2020) 41:1796-7. doi: 10.1093/eurheartj/ehaa301

22. Qi X, Liu C, Jiang Z, Gu Y, Zhang G, Shao C, et al. Multicenter analysis of clinical characteristics and outcome of COVID-19 patients with liver injury. $J$ Hepatol. (2020) 73:45-8. doi: 10.1016/j.jhep.2020.04.010

23. Cai Q, Huang D, Yu H, Zhu Z, Xia Z, Su Y, et al. Characteristics of liver tests in COVID-19 patients. J Hepatol. (2020) doi: 10.1016/j.jhep.2020.04.006. [Epub ahead of print].

24. Ren JL, Zhang AH, Wang XJ. Traditional Chinese medicine for COVID-19 treatment. Pharmacol Res. (2020) 155:104743. doi: 10.1016/j.phrs.2020.104743

25. Yang Y, Islam MS, Wang J, Li Y, Chen X. Traditional Chinese medicine in the treatment of patients infected with 2019-new Coronavirus (SARSCoV-2): a review and perspective. Int J Biol Sci. (2020) 16:170817. doi: $10.7150 /$ ijbs. 45538

26. Wan S, Yi Q, Fan S, Lv J, Zhang X, Guo L, et al. Characteristics of lymphocyte subsets and cytokines in peripheral blood of 123 hospitalized patients with 2019 novel coronavirus pneumonia (NCP). medRxiv. (2020) doi: 10.1101/2020.02.10.20021832. [Epub ahead of print].

27. Ye Q, Wang B, Mao J. Cytokine storm in COVID-19 and treatment. J Infect. (2020) 80:607-13. doi: 10.1016/j.jinf.2020.03.037

28. Chen L, Liu HG, Liu W, Liu J, Liu K, Shang J, et al. Analysis of clinical features of 29 patients with 2019 novel coronavirus pneumonia. Zhonghua Jie He He Hu Xi Za Zhi. (2020) 43:E005. doi: 10.3760/cma.j.issn.1001-0939.202 0.0005

29. Mehta P, McAuley DF, Brown M, Sanchez E, Tattersall RS, Manson JJ. COVID19: consider cytokine storm syndromes and immunosuppression. Lancet. (2020) 395:1033-4. doi: 10.1016/S0140-6736(20)30628-0

30. Henderson LA, Canna SW, Schulert GS, Volpi S, Lee PY, Kernan KF, et al. On the alert for cytokine storm: Immunopathology in COVID-19. Arthritis Rheumatol. (2020) 72:1059-63. doi: 10.1002/art.41285

31. Brigham KL. Lower tidal volume ventilation and plasma cytokine markers of inflammation in patients with acute lung injury. Curr Infect Dis Rep. (2005) 7:327-8. doi: 10.1007/s11908-005-0004-2

32. Lew TW, Kwek TK, Tai D, Earnest A, Loo S, Singh K, et al. G. Acute respiratory distress syndrome in critically ill patients with severe acute respiratory syndrome. JAMA. (2003) 290:374-80. doi: 10.1001/jama.290.3.374

33. Kim KD, Zhao J, Auh $\mathrm{S}$, Yang $\mathrm{X}$, Du P, Tang $\mathrm{H}$, et al. Adaptive immune cells temper initial innate responses. Nat Med. (2007) 13:124852. doi: $10.1038 / \mathrm{nm} 1633$

34. Zhao J, Zhao J, Perlman S. T cell responses are required for protection from clinical disease and for virus clearance in severe acute respiratory syndrome coronavirus-infected mice. J Virol. (2010) 84:9318-25. doi: 10.1128/JVI.01049-10

Conflict of Interest: The authors declare that the research was conducted in the absence of any commercial or financial relationships that could be construed as a potential conflict of interest.

Copyright (๑) 2020 Feng, Li, Ma, Liang, Lei, Li, Wang, Song, Li, Yang and Yang. This is an open-access article distributed under the terms of the Creative Commons Attribution License (CC BY). The use, distribution or reproduction in other forums is permitted, provided the original author(s) and the copyright owner(s) are credited and that the original publication in this journal is cited, in accordance with accepted academic practice. No use, distribution or reproduction is permitted which does not comply with these terms. 